\title{
SEMIGROUPS OF LEFT QUOTIENTS-THE UNIQUENESS PROBLEM
}

\author{
by VICTORIA GOULD
}

(Received 7th June 1990)

\begin{abstract}
Let $S$ be a subsemigroup of a semigroup $Q$. Then $Q$ is a semigroup of left quotients of $S$ if every element of $Q$ can be written as $a^{*} b$, where $a$ lies in a group $\mathscr{H}$-class of $Q$ and $a^{*}$ is the inverse of $a$ in this group; in addition, we insist that every element of $S$ satisfying a weak cancellation condition named square-cancellable lie in a subgroup of $Q$.

J. B. Fountain and $M$. Petrich gave an example of a semigroup having two non-isomorphic semigroups of left quotients. More positive results are available if we restrict the classes of semigroups from which the semigroups of left quotients may come. For example, a semigroup has at most one bisimple inverse $\omega$ semigroup of left quotients. The crux of the matter is the restrictions to a semigroup $S$ of Green's relations $\mathscr{R}$ and $\mathscr{L}$ in a semigroup of quotients of $S$. With this in mind we give necessary and sufficient conditions for two semigroups of left quotients of $S$ to be isomorphic under an isomorphism fixing $S$ pointwise.

The above result is then used to show that if $R$ is a subring of rings $Q_{1}$ and $Q_{2}$ and the multiplicative subsemigroups of $Q_{1}$ and $Q_{2}$ are semigroups of left quotients of the multiplicative semigroup of $R$, then $Q_{1}$ and $Q_{2}$ are isomorphic rings.
\end{abstract}

1980 Mathematics subject classification (1985 Revision): 20M

\section{Introduction}

This paper is concerned with the following question: if $Q_{1}$ and $Q_{2}$ are semigroups of left quotients of a semigroup $S$, under which conditions is $Q_{1}$ isomorphic to $Q_{2}$ under an isomorphism whose restriction to $S$ is the identity map? We call such an isomorphism an isomorphism over $S$. Our notion of a semigroup of left quotients is that introduced by Fountain and Petrich in [6] and developed in [7]; in Section 2 we give a precise definition, restricting ourselves here to remarking that the approach is via consideration of group inverses of elements. If $Q$ is a semigroup of left quotients of $S$ then we also say that $S$ is a left order in $Q$.

It is well known that if a ring $R$ has classical rings of left quotients $Q_{1}$ and $Q_{2}$, then $Q_{1}$ and $Q_{2}$ are isomorphic under a ring isomorphism over $R$ (Lambek [12]). Unfortunately, the corresponding result is not true for semigroups. In the first paper of this area, Fountain and Petrich [6] give an example of a semigroup having two nonisomorphic semigroups of left quotients. However, by restricting the class of semigroups of left quotients under consideration, one can obtain some positive results. For example, a semigroup has, up to isomorphism, at most one bisimple inverse $\omega$-semigroup of left quotients (Gould [8]). The reason for this is made clear by Theorem 3.1 of Gould [10], 
where the author gives sufficient conditions for two semigroups of left quotients of a semigroup $S$ to be isomorphic over $S$, in the special case where the semigroups of left quotients are of a particular kind, later called straight; moreover, these conditions are easily seen to be necessary. The given conditions involve binary relations on $S$ obtained by restricting Green's relations $\mathscr{L}$ and $\mathscr{R}$ in the semigroup of left quotients to $S$. Since every left order in a bisimple inverse $\omega$-semigroup must be straight, Theorem 3.1 of Gould [10] can be applied to yield the above-mentioned result that if $S$ is a left order in bisimple inverse $\omega$-semigroups $Q_{1}$ and $Q_{2}$, then $Q_{1}$ and $Q_{2}$ are isomorphic over $S$. Indeed its scope of application is far wider than this, for as shown in Gould [9], if $S$ is a left order in a regular semigroup $Q$ and $\mathscr{H}$ is a congruence on $Q$, then $S$ is straight in $Q$.

After some preliminaries in Section 2, we give in Section 3 the main result of this paper, which determines in the general case when two semigroups of left quotients of a given semigroup $S$ are isomorphic over $S$. In fact we prove the result for the more general concept of a weak left order. We make use of preorders on $S$ obtained by restricting the preorders $\leqq_{\mathscr{L}}$ and $\leqq_{\mathscr{t}}$ on a semigroup of left quotients to $S$. In addition we must take into account a ternary relation on $S$, which is again connected with Green's relations on a semigroup of left quotients.

Section 4 offers some corollaries to Theorem 3.1. We show that in some rather general cases we can dispense with mention of the ternary relation. In particular, this is so if the left order is straight, where we can improve upon Theorem 3.1 of Gould [10].

If $R$ is a subring of a ring $Q$ then we say that $R$ is a left order in $Q$ or $Q$ is a ring of left quotients of $R$ if the multiplicative semigroup of $R$ is a left order in the multiplicative semigroup of $Q$. There is no insistence here that $Q$ have an identity, but where it does, there is a surprisingly close connection with the notion of a classical ring of left quotients (Fountain and Gould $[3,4,5]$ ). One would hope, therefore, that any two rings of left quotients of a ring $R$ were isomorphic (as rings) over $R$. Theorem 5.5 of Fountain and Gould [3] says this is true if the rings of left quotients are (von Neumann) regular. In Section 5 we show that the results of this paper allow us to drop the restriction of regularity and prove that, up to isomorphism, a ring has at most one ring of left quotients.

\section{Preliminaries}

Where possible we follow standard notation and terminology, as may be found in Howie [11] and Clifford and Preston [1]. In particular, $E(S)$ always denotes the set of idempotents of a semigroup $S$.

An element $b$ of a semigroup $S$ is a group inverse of the element $a$ of $S$ if $a b a=a$, $b a b=b$ and $a b=b a$. A group inverse of $a \in S$, if it exists, is unique and is denoted by $a^{*}$. It is not difficult to see that $a^{*}$ exists if and only if $a$ lies in a group $\mathscr{H}$-class of $S$ and $a^{*}$ is the inverse of $a$ in $H_{a}$.

The relation $\mathscr{L}^{*}$ is defined on a semigroup $S$ by the rule that if $a, b \in S$ then $a \mathscr{L}^{*} b$ if for all $x, y \in S^{1}, a x=a y$ if and only if $b x=b y$. An alternative description of $\mathscr{L}^{*}$ is given in Fountain [2], namely, if $a, b \in S$ then $a \mathscr{L}^{*} b$ in $S$ if and only if $a \mathscr{L} b$ in some oversemigroup of $S$. The relation $\mathscr{R}^{*}$ is defined dually and $\mathscr{H}^{*}$ is the intersection of $\mathscr{L}^{*}$ 
and $\mathscr{R}^{*}$. Clearly on any semigroup $\mathscr{L} \subseteq \mathscr{L}^{*}, \mathscr{R} \subseteq \mathscr{R}^{*}$ and $\mathscr{H} \subseteq \mathscr{H}^{*}$ : if the semigroup is regular then it is straightforward to show that these inclusions are equalities. An element $a$ of $S$ is square-cancellable if $a \mathscr{H}^{*} a^{2}$-clearly this is a necessary condition for $a$ to have a group inverse in an oversemigroup of $S$. The set of square-cancellable elements of $S$ is denoted by $\mathscr{S}(S)$ and the set of elements lying in group $\mathscr{H}$-classes by $\mathscr{H}(S)$. If $S$ is a subsemigroup of $Q$ then $\mathscr{H}(Q) \cap S$ is denoted by $\mathscr{G}(Q, S)$.

We now give the precise definition of a semigroup of left quotients.

Let $S$ be a subsemigroup of a semigroup $Q$. Then $S$ is a left order in $Q$ and $Q$ is a semigroup of left quotients of $S$ if

(i) any $q$ can be written as $a^{*} b$ where $a, b \in S$, and

(ii) every square-cancellable element of $S$ has a group inverse in $Q$.

In the case where condition (i) alone holds, we say that $S$ is a weak left order in $Q$ and $Q$ is a semigroup of weak left quotients of $S$. If $S$ is a left order in $Q$ then $S$ is a straight left order in $Q$ if in addition every $q$ in $Q$ can be written as $a^{*} b$ where $a, b \in S$ and $a \mathscr{R} b$ in $Q$. These definitions have their natural left-right duals, yielding the notions of right order, etc. By dropping the adjective left or right from these definitions, we mean that both left and right conditions hold. For instance, $S$ is an order in $Q$ if $S$ is both a left order and a right order in $Q$.

Our approach is partially inspired by the notion of a classical left order in ring theory. Recall that a subring $R$ of a ring $Q$ is a classical left order in $Q$ if $Q$ has an identity, every $q$ in $Q$ can be written as $a^{-1} b$ where $a, b \in R$, and every non-zero divisor of $R$ has an inverse in $Q$. Certainly the notion of a left order in a semigroup is designed for semigroups where, roughly speaking, the behaviour of each of the subgroups is equally important. Nevertheless it is rather surprisingly closely connected with that of a classical left order in a ring, where the concentration is on the group of units. Further details may be found in Fountain and Gould [3]. With this in mind we apply our definitions of left order etc. to the multiplicative semigroups of rings, with the added restriction that if, for example, a ring $R$ is a left order in a ring $Q$, then we insist that $R$ is in addition a subring of $Q$.

Significant to our arguments are not just Green's relations $\mathscr{L}$ and $\mathscr{R}$ and their generalisations $\mathscr{L}^{*}$ and $\mathscr{R}^{*}$, but certain natural preorders with which they are the associated equivalences. These are denoted by $\leqq \mathscr{L}, \varliminf_{\mathscr{R}}, \varliminf_{\mathscr{*}}$ and $\varliminf_{\mathscr{R}^{*}}$ respectively and defined for elements $a, b$ of a semigroup $S$ by

$$
\begin{aligned}
& a \leqq_{\mathscr{P}} b \text { if and only if } S^{1} a \subseteq S^{1} b, \\
& a \leqq_{\mathfrak{R}} b \text { if and only if } a S^{1} \subseteq b S^{1}, \\
& a \leqq_{\mathscr{L}} b \text { if and only if for all } x, y \in S^{1}, b x=b y \text { implies that } a x=a y,
\end{aligned}
$$

and 
$a \leqq b$ if and only if for all $x, y \in S^{1}, x b=y b$ implies that $x a=y a$.

Clearly $\leqq_{\mathscr{L}} \subseteq \leqq_{\mathscr{L}^{*}}$ and $\leqq_{\mathscr{R}} \subseteq \leqq_{\mathscr{R}^{*}}$ and it is easy to see that if $S$ is regular then these inclusions are equalities. Further, the preorders $\leqq \mathscr{L}_{*}$ and $\leqq \mathscr{L}$ are compatible with multiplication on the right, forcing $\mathscr{L}^{*}$ and $\mathscr{L}$ to be right congruences on $S$. Dually, $\leqq_{\mathscr{R}^{*}}$ and $\leqq_{\mathscr{R}}$ are compatible with multiplication on the left, so that $\mathscr{R}^{*}$ and $\mathscr{R}$ are left congruences on $S$.

The following lemma will be used repeatedly. Its proof is very easy and so is omitted.

Lemma 2.1. Let $S$ be a weak left order in a semigroup $Q$. If $q \in Q$, then $q=a^{*} b$ for some $a \in \mathscr{G}(Q, S)$ and $b \in S$ with $b \leqq_{\mathfrak{R}} a$ in $Q$.

At this point we make a rather simple remark, but one that will be heavily relied upon without further comment. Suppose that in a semigroup $S$ we have an expression of the form $a q=p$ where $a \in \mathscr{H}(S), p, q \in S$ and $q \leqq a a$ in $S$. Then $q=a^{*} p$. For $q S^{1} \subseteq a S^{1}=$ $a^{*} a S^{1}, a^{*} a \in E(S)$ and so $q=a^{*} a q=a^{*} p$.

Let $S$ be a subsemigroup of $Q$. Then the restrictions to $S$ of the relations $\leqq_{\mathscr{L}}, \leqq_{\mathscr{R}}, \mathscr{L}$ and $\mathscr{R}$ on $Q$ are denoted by $\leqq_{1}, \leqq_{r}, \mathscr{L}^{\prime}$ and $\mathscr{R}^{\prime}$ respectively. We should more properly write $\leqq_{l}(Q, S), \leqq_{r}(Q, S), \mathscr{L}^{\prime}(Q, S)$ and $\mathscr{R}^{\prime}(Q, S)$, but we dispense with this clumsy notation by carefully avoiding any real possibility of ambiguity. Clearly $\leqq_{l} \subseteq \leqq_{\mathscr{L}^{*} \text {, }}$ $\leqq_{r} \subseteq \leqq_{\mathscr{R}^{*}}, \mathscr{L}^{\prime} \subseteq \mathscr{L}^{*}$ and $\mathscr{R}^{\prime} \subseteq \mathscr{R}^{*}$. The relations $\leqq_{l}$ and $\leqq_{r}$ are preorders with associated equivalences $\mathscr{L}^{\prime}$ and $\mathscr{R}^{\prime}$. Moreover $\leqq_{l}\left(\leqq_{r}\right)$ is right (left) compatible with multiplication, so that $\mathscr{L}^{\prime}\left(\mathscr{R}^{\prime}\right)$ is a right (left) congruence on $S$.

Finally in this section we define the ternary relation $\mathscr{T}(Q, S)$ on a subsemigroup $S$ of $Q$, mentioned in the introduction. Let $a, b, c \in S$. Then $(a, b, c) \in \mathscr{T}(Q, S)$ if and only if $a, c \in \mathscr{G}(Q, S), b \leqq_{\mathscr{R}} a$ in $Q$ and $a^{*} b Q^{1} \subseteq c Q^{1}$.

\section{The uniqueness theorem}

This section is devoted to the proof of the following theorem.

Theorem 3.1. Let $S$ be a weak left order in a semigroup $Q$ and $\phi: S \rightarrow P$ be an embedding of $S$ into a semigroup $P$ such that $S \phi$ is a weak left order in $P$. Then $Q$ is isomorphic to $P$ under an isomorphism $\bar{\phi}$ extending $\phi$ if and only if for all $a, b, c \in S$,

$$
(a, b, c) \in \mathscr{T}(Q, S) \text { if and only if }(a \phi, b \phi, c \phi) \in \mathscr{T}(P, S \phi) .
$$

Moreover, if this condition holds then $\bar{\phi}$ is unique.

Proof. Suppose first that there exists an isomorphism $\phi: Q \rightarrow P$ extending $\phi$. Clearly $\Phi$ is unique, for if $\psi: Q \rightarrow P$ is any isomorphism extending $\phi$ and $q=a^{*} b$ where $a, b \in S$, then $q \psi=\left(a^{*} b\right)=\left(a^{*}\right) \psi b \psi=(a \psi)^{*} b=\psi(a \phi)^{*} b \phi$.

Assume we are given $a, b, c \in S$ with $(a, b, c) \in \mathscr{T}(Q, S)$. By definition, $a, c$ are elements of $\mathscr{G}(Q, S), b Q^{1} \subseteq a Q^{1}$ and $a^{*} b Q^{1} \subseteq c Q^{1}$. Since $a$ and $c$ lie in subgroups of $Q$, certainly $a \phi$ and $c \phi$ lie in subgroups of $P$, that is, $a \phi, c \phi \in \mathscr{G}(P, S \phi)$. Further, $b \phi P^{1}=b \bar{\phi} P^{1} \subseteq a \bar{\phi} P^{1}=$ 
$a \phi P^{1}$ and $(a \phi)^{*} b \phi P^{1}=\left(a^{*} b\right) \phi P^{1} \subseteq c \phi P^{1}=c \phi P^{1}$ so that $(a \phi, b \phi, c \phi) \in \mathscr{T}(P, S \phi)$. The same argument applied to $\bar{\phi}^{-1}: S \phi \rightarrow S$ gives that if $(a \phi, b \phi, c \phi) \in \mathscr{T}(P, S \phi)$, then $(a, b, c) \in \mathscr{T}(Q, S)$.

Conversely we suppose that for all $a, b, c \in S,(a, b, c) \in \mathscr{T}(Q, S)$ if and only if $(a \phi, b \phi, c \phi) \in \mathscr{T}(P, S \phi)$.

Let $a \in S$. We claim that $a \in \mathscr{G}(Q, S)$ if and only if $a \phi \in \mathscr{G}(P, S \phi)$. For if $a \in \mathscr{G}(Q, S)$ then $(a, a, a) \in \mathscr{T}(Q, S)$ so that $(a \phi, a \phi, a \phi) \in \mathscr{T}(P, S \phi)$ and in particular, $a \phi \in \mathscr{G}(P, S \phi)$. Clearly the same argument can be applied to $\phi^{-1}$ to complete the proof of the claim.

We next show that if $a \in \mathscr{G}(Q, S)$ and $b \in S$, then $b Q^{1} \subseteq a Q^{1}$ if and only if $b \phi P^{1} \subseteq a \phi P^{1}$. Again, it is only necessary to prove this implication in one direction. Now if $b Q^{1} \subseteq a Q^{1}$ than $(a, b, a) \in \mathscr{T}(Q, S)$ so that by assumption $(a \phi, b \phi, a \phi) \in \mathscr{T}(P, S \phi)$ and then $b \phi P^{1} \subseteq$ $a \phi P^{1}$.

We now define $\phi: Q \rightarrow P$ by putting $\left(a^{*} b\right) \bar{\phi}=(a \phi)^{*} b \phi$ where $a, b \in S$ and $b \leqq_{r} a$. In view of Lemma 2.1, $\bar{\phi}$ is defined for every $q \in Q$.

Lemma 3.2. The function $\phi$ is well-defined and injective.

Proof. We show that $\bar{\phi}$ is well-defined, the proof that $\bar{\phi}$ is injective being dual.

Suppose now that $a, c \in \mathscr{G}(Q, S), b, d \in S, b \leqq_{r} a, d \leqq_{r} c$ and $a^{*} b=c^{*} d$. Certainly

$$
b \phi \leqq, a \phi
$$

and $(a, b, c) \in \mathscr{T}(Q, S)$, so that

$$
(a \phi, b \phi, c \phi) \in \mathscr{T}(P, S \phi)
$$

From $a^{*} b=c^{*} d$ we have that $c a^{*} b=d$; now express $c a^{*}$ as $c a^{*}=h^{*} k$ where $h \in \mathscr{G}(Q, S)$, $k \in S$ and $k \leqq_{r} h$. Then $h c a=k a^{2}$ and $c a \mathscr{R} c a^{*} \leqq_{\mathscr{R}} h$ in $Q$, so that

$$
c \phi a \phi \leqq, h \phi
$$

and

$$
h \phi c \phi a \phi=k \phi(a \phi)^{2}
$$

Further, $d=h^{*} k b$ gives that $d \leqq, h$ and $h d=k b$. Hence

$$
d \phi \leqq, h \phi
$$

and

$$
h \phi d \phi=k \phi b \phi .
$$

From (3.2.3) and (3.2.4) we deduce that $c \phi a \phi=(h \phi)^{*} k \phi(a \phi)^{2}$ and from (3.2.5) and (3.2.6) that $d \phi=(h \phi)^{*} k \phi b \phi$. Now using (3.2.1), we have 


$$
d \phi=(h \phi)^{*} k \phi(a \phi)^{2}(a \phi)^{*}(a \phi)^{*} b \phi=c \phi a \phi(a \phi)^{*}(a \phi)^{*} b \phi=c \phi(a \phi)^{*} b \phi
$$

Then (3.2.2) yields $(a \phi)^{*} b \phi=(c \phi)^{*} d \phi$. Consequently, $\Phi$ is well defined.

Lemma 3.3. The function $\Phi$ is a homomorphism.

Proof. Let $a^{*} b, c^{*} d \in Q$, where $a, c \in \mathscr{G}(Q, S), b, d \in S, b \leqq_{r} a$ and $d \leqq_{r} c$. As above, $a \phi, c \phi \in \mathscr{G}(P, S \phi)$ and

$$
b \phi \leqq, a \phi, d \phi \leqq r c \phi
$$

Choose $h \in \mathscr{G}(Q, S), k \in S$ with $k \leqq{ }_{\mathrm{r}} h$ and $a^{*} b c^{*}=h^{*} k$. Notice that $a^{*} b c \mathscr{R} a^{*} b c^{*} \leqq, h$ in $Q$ and $b c \leqq, b \leqq, a$ so that $(a, b c, h) \in \mathscr{T}(Q, S)$ and then

$$
(a \phi, b \phi c \phi, h \phi) \in \mathscr{T}(P, S \phi) .
$$

From $a^{*} b c^{*}=h^{*} k$ we have $h a^{*} b c=k c^{2}$ and we may then express $h a^{*}$ as $h a^{*}=u^{*} v$ where $u \in \mathscr{G}(Q, S), v \in S$ and $v \leqq_{\mathrm{r}} u$. One deduces that $h a \leqq_{\mathrm{r}} u$, $u h a=v a^{2}, k c^{2} \leqq_{\mathrm{r}} u$ and $u k c^{2}=v b c$. Hence in $P$,

$$
\begin{gathered}
h \phi a \phi \leqq \\
{ }_{r} u \phi \\
u \phi h \phi a \phi=v \phi(a \phi)^{2} \\
k \phi(c \phi)^{2} \leqq_{r} u \phi
\end{gathered}
$$

and

$$
u \phi k \phi(c \phi)^{2}=v \phi b \phi c \phi
$$

Expressions (3.3.3) and (3.3.4) yield $h \phi a \phi=(u \phi)^{*} v \phi(a \phi)^{2}$ and (3.3.5) and (3.3.6) yield

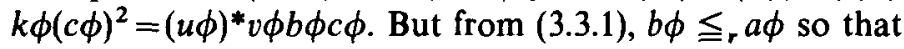

$$
k \phi(c \phi)^{2}=(u \phi)^{*} v \phi(a \phi)^{2}(a \phi)^{*}(a \phi)^{*} b \phi c \phi=h \phi a \phi(a \phi)^{*}(a \phi)^{*} b \phi c \phi=h \phi(a \phi)^{*} b \phi c \phi .
$$

Now (3.3.2) gives that $(h \phi)^{*} k \phi(c \phi)^{2}=(a \phi)^{*} b \phi c \phi$. We know from (3.3.1) that $d \phi \leqq_{r} c \phi$ and so

$$
(h \phi)^{*} k \phi d \phi=(h \phi)^{*} k \phi(c \phi)^{2}(c \phi)^{*}(c \phi)^{*} d \phi=(a \phi)^{*} b \phi c \phi(c \phi)^{*}(c \phi)^{*} d \phi=(a \phi)^{*} b \phi(c \phi)^{*} d \phi
$$

Certainly $k d \leqq_{r} k \leqq_{r} h$, so that

$$
\left(a^{*} b c^{*} d\right) \bar{\phi}=\left(h^{*} k d\right) \Phi=(h \phi)^{*}(k d) \phi=(a \phi)^{*} b \phi(c \phi)^{*} d \phi=\left(a^{*} b\right) \Phi\left(c^{*} d\right) \Phi
$$

therefore, $\Phi$ is a homomorphism.

To complete the proof that $\bar{\phi}$ is an isomorphism, we remark that as any $p \in P$ can be 
written as $p=(a \phi)^{*} b \phi$ with $a \phi \in \mathscr{G}(P, S \phi)$ and $b \phi \in S \phi$ where $b \phi \varliminf_{r} a \phi$, then since $a \in \mathscr{G}(Q, S)$ and $b \leqq, a$ we have that $p=\left(a^{*} b\right) \phi$. Thus $\phi$ is onto and hence an isomorphism.

Finally, to see that $\bar{\phi}$ extends $\phi$, let $s \in S$ and express $s$ as $s=a^{*} b$ where $a \in \mathscr{G}(Q, S)$, $b \in S$ and $b \varliminf_{r} a$. Then $a s=b$ and $s \varliminf_{\mathrm{r}} a$. Thus $s \phi \varliminf_{\mathrm{r}} a \phi$ and $s \phi=(a \phi)^{*} b \phi=(a \phi)^{*} a \phi s \phi=$ $s \phi$ as required.

\section{Some special cases}

We begin by commenting that if $S$ is a left order in a semigroup $Q$, then $\mathscr{G}(Q, S)=\mathscr{S}(S)$ and is independent of $Q$.

Attempting to avoid mention in Theorem 3.1 of the ternary relation $\mathscr{T}(Q, S)$ leads us to the following definition.

Let $S$ be a left order in a semigroup $Q$. Then $S$ is an $\mathscr{R}$-bounded left order in $Q$ if for all $q \in Q$ and $a, c \in \mathscr{S}(S)$, if $q \leqq_{\mathscr{R}} a, c$ in $Q$, then there is a $w \in S$ with $q \leqq_{\mathscr{R}} w \leqq_{\mathfrak{R}} a, c$. Many left orders are $\mathscr{R}$-bounded, as shown in Lemma 4.2. We first demonstrate that by restricting our attention to $\mathscr{R}$-bounded left orders we can replace the relation $\mathscr{T}(Q, S)$ in Theorem 4.1 by $\leqq$.

Proposition 4.1. Let $S$ be an $\mathscr{R}$-bounded left order in a semigroup $Q$ and $\phi: S \rightarrow P$ be an embedding of $S$ as an $\mathscr{R}$-bounded left order in a semigroup $P$. Then $Q$ is isomorphic to $P$ under an isomorphism $\bar{\phi}$ extending $\phi$ if and only if

$$
b \leqq_{r} d \text { if and only if } b \phi \leqq_{r} d \phi
$$

for all $b, d \in S$. Moreover, if this condition holds then $\Phi$ is unique.

Proof. If $\phi$ exists then it is clear that for all $b, d \in S, b \leqq_{\mathrm{r}} d$ if and only if $b \phi \varliminf_{\mathrm{r}} d \phi$. Conversely, suppose that this condition holds and $(a, b, c) \in \mathscr{T}(Q, S)$. Then $a, c \in \mathscr{S}(S)$, $b \leqq_{\mathrm{r}} a$ and $a^{*} b Q^{1} \subseteq c Q^{1}$. Certainly $a \phi, c \phi \in \mathscr{S}(S \phi)$ and by assumption $b \phi \leqq_{\mathrm{r}} a \phi$.

Now $S$ is $\mathscr{R}$-bounded in $Q$ and putting $q=a^{*} b, q \leqq a, c$ in $Q$ and $a, c \in \mathscr{S}(S)$. Thus there is an element $w$ of $S$ with $q \leqq a w \leqq a, c$ in $Q$. Hence $b \leqq_{r} a w$ and $w \leqq_{r} a, c$ in $S$, giving that in $S \phi, b \phi \leqq, a \phi w \phi$ and $w \phi \leqq, a \phi, c \phi$. We can now deduce

$$
(a \phi)^{*} b \phi P^{1} \subseteq(a \phi)^{*} a \phi w \phi P^{1}=(w \phi) P^{1} \subseteq c \phi P^{1}
$$

so that $(a \phi, b \phi, c \phi) \in \mathscr{T}(P, S \phi)$. Dually, if $(a \phi, b \phi, c \phi) \in \mathscr{T}(P, S \phi)$, then $(a, b, c) \in \mathscr{T}(Q, S)$. Theorem 3.1 can now be applied to guarantee the existence of a unique isomorphism $\Phi: Q \rightarrow P$ extending $\phi$.

We now give the promised examples of $\mathscr{R}$-bounded left orders.

Lemma 4.2. Let $S$ be a left order in $Q$. Then $S$ is $R$-bounded if any of the following conditions hold. 
(i) $S$ intersects every $\mathscr{R}$-class of $Q$.

(ii) $S$ is straight in $Q$.

(iii) $S$ is an order in $Q$.

Proof. It is immediate that if $S$ intersects every $\mathscr{R}$-class of $Q$, then $S$ is $\mathscr{R}$-bounded in $Q$. By Proposition 4 of Gould [9], if $S$ is straight in $Q$ then $S$ intersects every $\mathscr{H}$-class of $Q$ and so certainly every $\mathscr{R}$-class. Finally, if $S$ is an order in $Q$ and $R$ is an $\mathscr{R}$-class of $Q$, then choosing $q \in R$ we can write $q=b a^{*}$ where $b \in S$ and $a \in \mathscr{S}(S)$. Then $q=b a^{*} \mathscr{R} b a$ in $Q$, giving that $b a \in R \cap S$ and (i) holds.

If $S$ intersects every $\mathscr{R}$-class of its semigroup of left quotients, then Proposition 4.1 can be simplified yet further.

Corollary 4.3. Let $S$ be a left order in a semigroup $Q$ and $\phi: S \rightarrow P$ be an embedding of $S$ as a left order in a semigroup $P$. Suppose further that $S$ intersects every $\mathscr{R}$-class of $Q$ and $S \phi$ intersects every $\mathscr{R}$-class of $P$. Then $Q$ is isomorphic to $P$ under an isomorphism $\bar{\phi}$ extending $\phi$ if and only if

\section{$b \mathscr{R}^{\prime} d$ if and only if $\quad b \phi \mathscr{R}^{\prime} d \phi$}

for all $b, d \in S$. Moreover, if this condition holds then $\bar{\phi}$ is unique.

Proof. By Lemma 4.2, $S$ is $\mathscr{R}$-bounded in $Q$ and $S \phi$ is $\mathscr{R}$-bounded in $P$. Proposition 4.1 now says that $\bar{\phi}$ exists if and only if

$$
b \leqq{ }_{r} d \text { if and only if } b \phi \leqq, d \phi
$$

for all $b, d \in S$. Thus if $\bar{\phi}$ exists, certainly

\section{$b \mathscr{R}^{\prime} d \quad$ if and only if $b \phi \mathscr{R}^{\prime} d \phi$}

for all $b, d \in S$.

Conversely, suppose that for all $b, d \in S$,

$$
b \mathscr{R}^{\prime} d \quad \text { if and only if } b \phi \mathscr{R}^{\prime} d \phi .
$$

Let $x, y \in S$ with $x \leqq r y$. Then $x Q^{1} \subseteq y Q^{1}$ so that $x=y q$ for some $q \in Q^{1}$. If $q=1$ then certainly $x \phi \leqq_{r} y \phi$. Assume therefore that $q \in Q$. Since $S$ intersects every $\mathscr{R}$-class of $Q$ we can choose $z \in R_{q} \cap S$ and then $x=y q \mathscr{R} y z$ in $Q$, so that $x \mathscr{R}^{\prime} y z$ in $S$. Hence $x \phi \mathscr{R}^{\prime} y \phi z \phi$ in $S \phi$ and then $x \phi P^{1}=y \phi z \phi P^{1} \subseteq y \phi P^{1}$ so that $x \phi \leqq{ }_{r} y \phi$ in $S \phi$. Conversely, from $u \phi \leqq r v \phi$ we deduce $u \leqq r v$. Thus by Proposition 4.1, $\phi$ exists as required.

We remark that Corollary 4.3 may certainly be applied to straight left orders, yielding a result which is a considerable improvement upon Theorem 3.1 of Gould [10]. 


\section{Left orders in rings}

We recall from Section 2 that if $R$ and $Q$ are rings then $R$ is a left order in $Q$ and $Q$ is a ring of left quotients of $R$ if $R$ is a subring of $Q$ and the multiplicative semigroup of $R$ is a left order in the multiplicative semigroup of $Q$. The aim of this section is to show that if a ring $R$ is a left order in rings $Q_{1}$ and $Q_{2}$, then $Q_{1}$ and $Q_{2}$ are isomorphic (as rings) over $R$. We first show that the relation $\mathscr{T}\left(Q_{i}, R\right)$ is independent of $i$, so that $\mathscr{T}\left(Q_{1}, R\right)=\mathscr{T}\left(Q_{2}, R\right)$. Theorem 3.1 gives immediately that $Q_{1}$ and $Q_{2}$ are isomorphic as multiplicative semigroups; some further work is required to prove they are isomorphic as rings.

If $a$ is an element of a ring $R$ then $a R^{1}$ denotes the principal right (multiplicative) semigroup ideal generated by $a$. Let $X$ be a subset of $R$. Then the left annihilator of $X$ in $R$, denoted by $l_{R}(X)$, is defined by

$$
l_{R}(X)=\{r \in R: r X=0\} .
$$

When $X=\{a\}$ is a singleton, we write $l_{R}(\{a\})$ more simply as $l_{R}(a)$. Right annihilators are defined dually. Then $R$ is right faithful if $r_{R}(R)=\{0\}$.

We make the convention that in the remainder of this section $R$ always denotes a subring of a ring $Q$.

Lemma 5.1. Let $R$ be a weak left order in $Q$. Then $R$ and $Q$ are right faithful.

Proof. Let $q \in Q$ and suppose that $R q=0$. By Lemma 2.1, $q=h^{*} k$ where $h \in \mathscr{G}(Q, R)$, $k \in R$ and $h h^{*} k=k$. Thus $0=h q=k$ and so $q=h^{*} 0=0$, giving that both $Q$ and $R$ are right faithful.

Corollary 5.2. Let $R$ be a weak left order in $Q$. Then the following are equivalent for elements $a, b$ of $R$ :

(i) $a \leqq a^{*} b$ in $R$;

(ii) $l_{R}(b) \subseteq l_{R}(a)$;

(iii) $l_{Q}(b) \subseteq l_{Q}(a)$;

(iv) $a \leqq a^{*} b$ in $Q$.

Proof. In view of Lemma 5.1, this is an immediate consequence of Lemma 2.7 of Fountain and Gould [3]. However, for the sake of completeness, we give the proof here.

(i) $\Rightarrow$ (ii) Suppose that $a \leqq a^{*} b$ in $R$ and $x \in l_{R}(b)$. Then $x b=0=0 b$ so as $a \leqq a^{*} b$, $x a=0 a=0$. Thus $x \in l_{R}(a)$, giving that $l_{R}(b) \subseteq l_{R}(a)$.

(ii) $\Rightarrow$ (iii) Given that $l_{R}(b) \subseteq l_{R}(a)$, suppose that $q \in l_{Q}(b)$. Then there exists $c \in \mathscr{G}(Q, R)$ and $d \in R$ with $d Q^{1} \subseteq c Q^{1}$ and $q=c^{*} d$. Then $d b=c c^{*} d b=c q b=c 0=0$, so that $d \in l_{R}(b)$, therefore $d a=0$ and so $q a=c^{*} d a=c^{*} 0=0$. Thus $l_{Q}(b) \subseteq l_{Q}(a)$.

(iii) $\Rightarrow$ (iv) Assume that $l_{Q}(b) \subseteq l_{Q}(a)$. Let $x, y \in Q^{1}$ and suppose that $x b=y b$. Hence, for 
any $q \in Q, q x b=q y b$, giving that $q x-q y \in l_{Q}(b) \subseteq l_{Q}(a)$, so that $q x a=q y a$ for all $q \in Q$. This in turn yields that $x a-y a \in r_{Q}(Q)$, but $r_{Q}(Q)=0$ since by Lemma $5.1, Q$ is right faithful. Thus $x a=y a$ and $a \leqq \mathscr{R}^{*} b$ in $Q$.

That (iv) implies (i) is clear.

Corollary 5.3. Let $R$ be a weak left order in $Q$. Let $a \in R$ and $h \in \mathscr{G}(Q, R)$. Then

$$
a \leqq h \text { if and only if } a \leqq \mathfrak{Q}^{*} h \text { in } R \text {. }
$$

Proof. Suppose that $a \leqq \mathscr{R}^{*} h$ in $R$. By Corollary 5.2, $a \leqq \mathscr{R}^{*} h$ in $Q$ so that $h=h h^{*} h$ implies $a=h h^{*} a$. Thus $a Q^{1}=h h^{*} Q^{1} \subseteq h Q^{1}$ and $a \leqq h$. As remarked in Section 2, the other implication always holds.

Corollary 5.3. is the first step to showing that if $R$ is a left order in $Q$, then the relation $\mathscr{T}(Q, R)$ is independent of $Q$. To smooth our further progress we make a new definition: a ring $Q$ is upper right regular if given any $q \in Q$ there is an $e \in E(Q)$ with $q \leqq \mathfrak{R} e$.

Lemma 5.4. Let $R$ be a weak left order in $Q$. Then $Q$ is upper right regular.

Proof. Let $q \in Q$. Then $q=h^{*} k$ for some $h \in \mathscr{G}(Q, R)$ and $k \in R$. Now $q Q^{1}=h^{*} k Q^{1} \subseteq$ $h^{*} Q^{1}=h h^{*} Q^{1}$, so that $q \leqq_{\mathscr{R}} e$ where $e=h h^{*} \in E(Q)$.

Upper right regular rings enjoy a property analogous to that of regular rings. Namely:

Proposition 5.5. Let $Q$ be an upper right regular ring. Then for all $x, y \in Q$, there exists an element $g$ of $E(Q)$ with $x \leqq_{\mathfrak{R}} g$ and $y \leqq_{\mathfrak{R}} g$.

Proof. Let $x, y \in Q$. Since $Q$ is upper right regular, there is an $e \in E(Q)$ with $x \leqq g$ and then an $f \in E(Q)$ with $y-e y \leqq a f$. Let $w=e+f-f e$. Then

$$
w x=(e+f-f e) x=x+f x-f x=x
$$

and

$$
w y=(e+f-f e) y=e y+f y-f e y=e y+f(y-e y)=e y+y-e y=y
$$

so that $x \leqq_{\mathfrak{R}} w$ and $y \leqq_{\mathfrak{R}} w$. Choosing $g \in E(Q)$ with $w \leqq_{\mathfrak{R}} g$ gives $x \leqq_{\mathfrak{R}} g$ and $y \leqq_{\mathfrak{x}} g$ as required.

The following proposition is crucial in that it allows us sufficient freedom of expression of elements in rings of left quotients in terms of elements of the left order. 
Proposition 5.6. Let $R$ be a weak left order in $Q$. Let $q \in Q$ and $r \in R$. Then $q=u^{*} v$ for some $u \in \mathscr{G}(Q, R)$ and $v \in R$ with $v \leqq_{r} u$ and $r \leqq{ }_{r} u$.

Proof. Certainly we can write $q$ as $q=a^{*} b$ for some $a \in \mathscr{G}(Q, R)$ and $b \in R$ with $b \leqq, a$. By Lemma 5.4, $Q$ is upper right regular and so by Proposition 5.5 there is an idempotent $g$ in $Q$ with $r \leqq_{q} g$ and $a \leqq_{q} g$ in $Q$. Now $g=c^{*} d$ for some $c \in \mathscr{G}(Q, R)$ and $d \in R$ with $d \leqq_{r} c$. Note also that $r \leqq_{r} c$ and $a \leqq_{r} c$. Put $e=c c^{*}, f=a a^{*}, \alpha=e-f e+a^{*} e$ and $\beta=e-f e+a e$. Straightforward calculation yields that $e \alpha=\alpha=\alpha e$ and $\alpha \beta=e=\beta \alpha$ so that $e \mathscr{H} \alpha$ in $Q$. Further,

$$
\alpha b=\left(e-f e+a^{*} e\right) b=a^{*} b=q
$$

and $\alpha=u^{*} v^{\prime}$ for some $u \in \mathscr{G}(Q, R)$ and $v^{\prime} \in R$ with $v^{\prime} \leqq r u$. Thus $q=u^{*} v$ where $v=v^{\prime} b$ and $v \leqq, u$. Finally, $r \leqq_{\mathscr{R}} c \mathscr{H} e \mathscr{H} \alpha \leqq_{\mathscr{R}} u$ in $Q$ so that $r \leqq_{r} u$ in $R$.

Corollary 5.7. Let $R$ be a weak left order in $Q$. Let $q \in Q$ and $r_{1}, r_{2} \in R$. Then $q=u^{*} v$ for some $u \in \mathscr{G}(Q, R)$ and $v \in R$ with $v \leqq, u$ and $r_{1}, r_{2} \leqq, u$.

Proof. It follows from Proposition 5.5 that there is an element $r \in R$ with $r_{1}, r_{2} \leqq r$. Using Proposition 5.6, the result is immediate.

Proposition 5.8. Let $R$ be a weak left order in $Q$. Let $a, c \in \mathscr{G}(Q, R)$ and $b \in R$ with $b \leqq, a$. Then $(a, b, c) \in \mathscr{T}(Q, R)$ if and only if there are elements $h, u, p \in \mathscr{G}(Q, R)$ and $k, v, q \in R$ with $k \leqq_{r} h, c h \varliminf_{r} u, a \varliminf_{r} u, u a \varliminf_{r} p, v k \leqq, p, u c h=v h^{2}, p u a=q a^{2}$ and $p v k=q b$.

Proof. Suppose first that $(a, b, c) \in \mathscr{T}(Q, R)$. Then, bearing in mind that $c Q^{1}=c Q$, we have that $a^{*} b=c h^{*} k$ for some $h \in \mathscr{G}(Q, R)$ and $k \in R$ with $k \leqq h$. Proposition 5.6 allows us to express $c h^{*}$ as $c h^{*}=u^{*} v$ where $u \in \mathscr{G}(Q, R), v \in R$ and $v, a \leqq r u$. Note also that $c h \leqq, u$ and from $c h^{*}=u^{*} v$ we deduce $u c h=v h^{2}$.

The equation $a^{*} b=c h^{*} k$ yields $a^{*} b=u^{*} v k$ and hence $u a^{*} b=v k$. Then $u a^{*}$ can be written as $u a^{*}=p^{*} q$ where $p \in \mathscr{G}(Q, R), q \in R$ and $q \varliminf_{r} p$. Then $u a \leqq_{r} p, p u a=q a^{2}$ and $v k=p^{*} q b$. Thus $v k \leqq_{r} p$ and $p v k=q b$.

Conversely, suppose that the elements $h, u, p, k, v, q$ exist satisfying the given conditions. From $u a, v k \leqq_{r} p$ and $p u a=q a^{2}, p v k=q b$, we have that $u a=p^{*} q a^{2}$ and $v k=p^{*} q b$. But $b \leqq_{r} a$ so that $v k=p^{*} q a^{2} a^{*} a^{*} b=u a a^{*} a^{*} b=u a^{*} b$. Now $c h \leqq, u$ and $u c h=v h^{2}$ gives that $c h=u^{*} v h^{2}$. Further, from $a \leqq, u$ we have that $u^{*} v k=a^{*} b$. Then

$$
a^{*} b=u^{*} v k=u^{*} v h^{2} h^{*} h^{*} k=\operatorname{ch} h^{*} h^{*} k=c h^{*} k
$$

so that $(a, b, c) \in \mathscr{T}(Q, R)$ as required.

The preceding result enables us to apply Theorem 3.1 to obtain the first part of the theorem below. 
Theorem 5.9. Let $R$ be a ring which is a left order in a ring $Q$, and let $\phi: R \rightarrow P$ be an embedding of $R$ as a left order in a ring $P$. Then $Q$ is isomorphic to $P$ under a unique isomorphism $\bar{\phi}$ extending $\phi$.

Proof. Let $a, b, c \in R$ and suppose that $(a, b, c) \in \mathscr{T}(Q, R)$. Then $a, c \in \mathscr{G}(Q, R)=\mathscr{S}(R)$, $b \leqq_{r} a$ and $a^{*} b Q^{1} \subseteq c Q^{1}$. Clearly $a \phi, c \phi \in \mathscr{S}(R \phi)=\mathscr{G}(P, R \phi)$ and Corollary 5.3 gives that $b \phi \leqq_{r} a \phi$. Then Proposition 5.8 can be applied to yield that $(a \phi, b \phi, c \phi) \in \mathscr{T}(P, R \phi)$. Similarly, if $(a \phi, b \phi, c \phi) \in \mathscr{T}(P, R \phi)$, then $(a, b, c) \in \mathscr{T}(Q, R)$. Theorem 3.1 then gives that $\phi$ can be extended to a unique semigroup isomorphism $\phi: Q \rightarrow P$. If we can show that $\bar{\phi}$ is also a ring homomorphism, then certainly $\bar{\phi}$ is the unique ring isomorphism from $Q$ to $P$ extending $\phi$.

Suppose now that $q_{1}=a^{*} b, q_{2}=c^{*} d$ are elements of $Q$ with $a, c \in \mathscr{S}(R), b, d \in R, b \varliminf_{r} a$ and $d \leqq r$. Then in $R \phi$,

$$
b \phi \leqq_{r} a \phi, d \phi \leqq_{r} c \phi .
$$

Using Corollary 5.7 we can write $q_{1}+q_{2}$ as $q_{1}+q_{2}=u^{*} v$ where $u \in \mathscr{S}(R), v \in R, v \leqq r u$, $a \leqq_{\mathrm{r}} u$ and $c \leqq_{\mathrm{r}} u$. Multiplying by $u$ gives that $v=u a^{*} b+u c^{*} d$. Further,

$$
a \phi \leqq r u \phi, \quad c \phi \leqq, u \phi .
$$

Now $u a^{*}=h^{*} k$ for some $h \in \mathscr{S}(R), k \in R$ with $k \leqq, h$. Then $u c^{*}=p^{*} q$ for some $p \in \mathscr{S}(R)$, $q \in R$ with $q \leqq_{\mathrm{r}} p$ and $h \leqq_{r} p$. Note also that $u a \leqq_{r} h, h u a=k a^{2}, u c \leqq_{\mathrm{r}} p$ and $p u c=q c^{2}$. Thus in $R \phi$,

$$
\begin{gathered}
k \phi \leqq_{r} h \phi, \quad q \phi \leqq_{r} p \phi, \quad h \phi \leqq_{r} p \phi, \\
u \phi a \phi \varliminf_{r} h \phi, \quad u \phi c \phi \leqq_{r} p \phi
\end{gathered}
$$

and

$$
h \phi u \phi a \phi=k \phi(a \phi)^{2}, \quad p \phi u \phi c \phi=q \phi(c \phi)^{2} .
$$

In addition we have that

$$
v=h^{*} k b+p^{*} q d
$$

so that $v Q^{1} \subseteq h^{*} k b Q^{1}+p^{*} q d Q^{1} \subseteq h Q^{1}+p Q^{1} \subseteq p Q^{1}+p Q^{1}=p Q^{1}$, giving

$$
v \phi \leqq r p \phi
$$

Multiplying in (5.9.6) by $p$ gives

$$
p v=p h^{*} k b+q d
$$

and so $p v-q d=p h^{*} k b$. Applying $\bar{\phi}$ we obtain 


$$
\begin{aligned}
(p v) \phi-(q d) \phi & =(p v-q d) \phi=(p v-q d) \phi=\left(p h^{*} k b\right) \Phi \\
& =p \bar{\phi}\left(h^{*}\right) \phi k \phi b \bar{\phi}=p \phi(h \phi)^{*} k \phi b \phi
\end{aligned}
$$

and so

$$
p \phi v \phi=p \phi(h \phi)^{*} k \phi b \phi+q \phi d \phi .
$$

From (5.9.3) and (5.9.7), $h \phi, v \phi \varliminf_{r} p \phi$ so that (5.9.8) gives

$$
v \phi=(h \phi)^{*} k \phi b \phi+(p \phi)^{*} q \phi d \phi .
$$

Using (5.9.4) and (5.9.5), $u \phi a \phi=(h \phi)^{*} k \phi(a \phi)^{2}$ so that from (5.9.1),

$$
\begin{aligned}
(h \phi)^{*} k \phi b \phi & =(h \phi)^{*} k \phi(a \phi)^{2}(a \phi)^{*}(a \phi)^{*} b \phi=u \phi a \phi(a \phi)^{*}(a \phi)^{*} b \phi \\
& =u \phi(a \phi)^{*} b \phi .
\end{aligned}
$$

Similarly, $(p \phi)^{*} q \phi d \phi=u \phi(c \phi)^{*} d \phi$. Returning now to (5.9.9), we have that

$$
v \phi=u \phi(a \phi)^{*} b \phi+u \phi(c \phi)^{*} d \phi .
$$

Applying (5.9.2) gives

$$
\left(q_{1}+q_{2}\right) \bar{\phi}=\left(u^{*} v\right) \phi=(u \phi)^{*} v \phi=(a \phi)^{*} b \phi+(c \phi)^{*} d \phi=\left(a^{*} b\right) \phi+\left(c^{*} d\right) \bar{\phi}
$$

which is $q_{1} \phi+q_{2} \phi$. Thus $\Phi$ is a ring homomorphism and the proof of Theorem 5.9 is complete.

\section{REFERENCES}

1. A. H. Cuifford and G. B. Preston, The Algebraic Theory of Semigroups (Math. Surveys 7, Vols. I and II, American Math. Soc. 1961, 1967).

2. J. B. Fountain, Abundant semigroups, Proc. London Math. Soc. 44 (1982), 103-129.

3. J. B. Fountarn and V. A. R. Gould, Orders in rings without identity, Preprint.

4. J. B. Fountain and V. A. R. Gould, Straight left orders in rings, Preprint.

5. J. B. Fountain and V. A. R. Gould, Orders in regular rings with minimal condition for principal right ideals, Preprint.

6. J. B. Fountain and M. Petrich, Completely 0-simple semigroups of quotients, J. Algebra 101 (1986), 365-402.

7. V. A. R. Gould, Semigroups of Quotients (D.Phil. thesis, University of York, 1985).

8. V. A. R. Gould, Bisimple inverse $\omega$-semigroups of left quotients, Proc. London Math. Soc. 52 (1986), 95-118. 
9. V. A. R. Gould, Orders in Semigroups (Contributions to General Algebra 5, Holder-PichlerTempsky, Wien, 1987).

10. V. A. R. Gould, Semigroups of Quotients (Proceedings of the International Symposium on the Theory of Regular Semigroups and Applications, University of Kerala, 1986).

11. J. M. Howie, An Introduction to Semigroup Theory (Academic Press, 1976).

12. J. LAMBEK, Lectures on Rings and Modules (Blaisdell Publishing Co., 1966).

Department of Mathematics

UNIVERSITY OF YORK

HESLINGTON

YoRK, YO1 5DD 